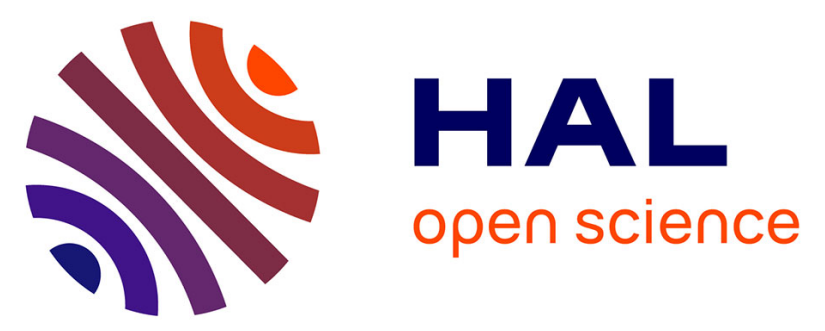

\title{
IMSYC IMmunologic SYnovitis sCore: A New Score for Synovial Membrane Characterization In Inflammatory And Non-Inflammatory Arthritis
}

Aurelie Najm, Benoit Le Goff, Guillaume Venet, Thomas Garraud, Jérôme Amiaud, Noura Biha, Céline Charrier, Sophie Touchais, Vincent Crenn, Frédéric Blanchard, et al.

\section{To cite this version:}

Aurelie Najm, Benoit Le Goff, Guillaume Venet, Thomas Garraud, Jérôme Amiaud, et al.. IMSYC IMmunologic SYnovitis sCore: A New Score for Synovial Membrane Characterization In Inflammatory And Non-Inflammatory Arthritis. Joint Bone Spine, 2018, Epub ahead of print. 10.1016/j.jbspin.2018.04.004 . inserm-01792046

\section{HAL Id: inserm-01792046 https://www.hal.inserm.fr/inserm-01792046}

Submitted on 15 May 2018

HAL is a multi-disciplinary open access archive for the deposit and dissemination of scientific research documents, whether they are published or not. The documents may come from teaching and research institutions in France or abroad, or from public or private research centers.
L'archive ouverte pluridisciplinaire HAL, est destinée au dépôt et à la diffusion de documents scientifiques de niveau recherche, publiés ou non, émanant des établissements d'enseignement et de recherche français ou étrangers, des laboratoires publics ou privés. 


\section{Accepted Manuscript}

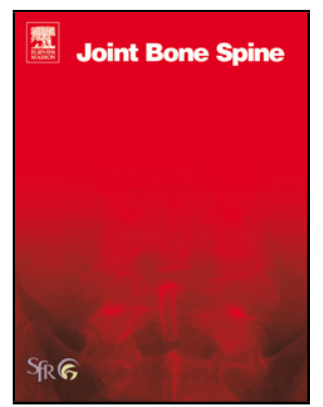

Title: IMSYC IMmunologic SYnovitis sCore: A New Score for Synovial Membrane Characterization In Inflammatory And Non-Inflammatory Arthritis

Author: Aurélie Najm Benoît le Goff Guillaume Venet Thomas Garraud Jérôme Amiaud Noura Biha Céline Charrier Sophie Touchais Vincent Crenn Frédéric Blanchard Veit Krenn

PII:

DOI:

S1297-319X(18)30084-8

Reference: https://doi.org/doi:10.1016/j.jbspin.2018.04.004 BONSOI 4722

To appear in:

Received date: 6-2-2018

Accepted date: $\quad$ 18-4-2018

Please cite this article as: Najm A, le Goff B, Venet G, Garraud T, Amiaud J, Biha N, Charrier C, Touchais S, Crenn V, Blanchard F, Krenn V, IMSYC IMmunologic SYnovitis sCore: A New Score for Synovial Membrane Characterization In Inflammatory And Non-Inflammatory Arthritis, Joint Bone Spine (2018), https://doi.org/10.1016/j.jbspin.2018.04.004

This is a PDF file of an unedited manuscript that has been accepted for publication. As a service to our customers we are providing this early version of the manuscript. The manuscript will undergo copyediting, typesetting, and review of the resulting proof before it is published in its final form. Please note that during the production process errors may be discovered which could affect the content, and all legal disclaimers that apply to the journal pertain. 
IMSYC IMmunologic SYnovitis sCore: A New Score for Synovial Membrane Characterization In Inflammatory And Non-Inflammatory Arthritis.

Aurélie Najm ${ }^{1,2}$, Benoît le Goff ${ }^{1,2}$, Guillaume Venet ${ }^{3}$, Thomas Garraud ${ }^{1,2}$, Jérôme Amiaud ${ }^{2}$, Noura Biha ${ }^{2}$, Céline Charrier ${ }^{2}$, Sophie Touchais ${ }^{4}$, Vincent Crenn ${ }^{4}$, Frédéric Blanchard ${ }^{2}$, Veit Krenn ${ }^{5}$.

1. Rheumatology Department, Nantes University Hospital, 44093 Nantes, France

2. INSERM UMR 1238 Laboratory, Phy-Os, 44093 Nantes, France

3. Orthopaedic surgery Department, La Roche sur Yon Hospital, 85925 La Roche sur Yon cedex 9, France

4. Orthopaedic surgery Department, Nantes University Hospital, 44093 Nantes, France

5. MVZ-Zentrum für Histologie, Zytologie und Molekulare Diagnostik, 54296 Trier,

Germany.

Corresponding Author: Doctor Aurélie Najm, Hôtel-Dieu Hospital, 1, place Alexis-

Ricordeau, 44093 Nantes cedex 1, France.

Tel: +33240084821

Fax: +33240084830

E-mail: aurelie.najm@gmail.com

\section{Abstract}

Objectives: Krenn synovitis Score has been developed by Krenn et al. in order to assess synovitis severity and is used in synovial research. Cell signature of synovial tissue can be studied using immunohistochemistry and is of interest as a biomarker for both prognosis and prediction of response to treatment. However, no synovitis score including

immunohistochemistry exists yet. In order to answer this unmet need, we propose a new Immunologic SYnovitis sCore (IMSYC) adding 5 components to the Krenn score: CD68, CD3, CD20, CD31 and Ki67 immunostaining. In this study, we aimed to validate this new IMSYC by studying its diagnostic performances in a well-defined collection of synovial samples.

Methods: Synovial samples from patients were obtained during surgical procedures. CD68, CD3, CD20, CD31 and KI67 immunohistochemistry were performed.

Results: 77 patients were included. 45 were females, mean age was 63.1 years. 40 had inflammatory arthritis, mainly rheumatoid arthritis (31/40). Non inflammatory arthritis group included 35 patients with mainly osteoarthritis. Mean Krenn score and IMSYC were significantly higher in the inflammatory group $(\mathrm{p}<0.001)$. ROC analysis of diagnostic performances determined the score of 13.5 out of 24 as the cut-off that gave the best ratio for discrimination between inflammatory and non-inflammatory arthritis with a sensitivity of $71.8 \%$ and specificity of $98 \%$.

Conclusion: We propose a new synovitis score including immunohistochemistry. This score has a better sensitivity and specificity than the Krenn score and represents a more functional 
synovitis evaluation. IMSYC could be further used in better categorizing synovial tissue phenotype and give a basis for tissue driven therapy.

Key words: Rheumatoid arthritis, Synovitis, Inflammation, Arthritis, Immunohistochemistry, Biomarker.

\section{Introduction}

Interest among synovial biopsies has expanded over the past few years, with development of minimally invasive techniques [1]. Synovial biopsies are more and more widely used in clinical setting for diagnostic purposes [2] and translational research setting. Moreover, the use of synovial membrane as a biomarker in rheumatic diseases (RMDs), especially in rheumatoid arthritis (RA) is a current area of intense research [3]. Immunohistochemistry (IHC) is frequently used for a better and more functional characterization of synovitis, and study of synovial membrane at a CD level can give valuable information. Pitzalis and colleagues have described different synovial pathotypes among RA patients based on cell signatures, studied with immunohistochemistry (IHC) [4]. Those pathotypes are associated with molecular signatures [5] and are highly suspected to have a close relationship with the clinical course of the disease, prognosis and also with therapeutic response or resistance to treatment. Indeed, it is highly probable that synovial tissue analysis at a cellular and molecular level will be a key element for personalized therapy in RA in the next few years.

Cellular analysis of synovitis can be performed by histological analysis. Krenn synovitis score (KS) has been developed by Krenn et al. in 2006 in order to quantify inflammation by scoring 3 major components of synovitis: lining layer hyperplasia, activation of resident cells (stroma) and inflammatory infiltrate. All components are graded semi-quantitatively from 0 to 3 with a maximum score of $9[6,7]$. In the original publication, KS allowed to discriminate low-grade and high-grade synovitis with a cut-off score of 5 out of 9 with a sensitivity of $61.7 \%$ and a specificity of $96.1 \%$. Following this first validation, this score has been internationally accepted and used over the past years in translational research for synovitis scoring. This is reflected by a high number of citations [8]. However, to perform a more accurate evaluation of synovitis at the cellular level, adding immunohistochemistry would be a valuable approach. Indeed, a synovitis score including immunohistochemistry is an unmet in the rheumatology field, especially for translational research.

In this work, we propose to create a new IMmunologic SYnovitis sCore (IMSYC) adding 5 CD components to the Krenn score: CD68 (macrophages), CD3 (T lymphocytes), CD20 (B lymphocytes), CD31 (endothelial cells) and Ki67 (proliferating cell) immunostaining. In order to validate this score, we first aimed to assess its diagnostic performances in a well-defined clinical collection of synovial samples.

The objectives of this work were to evaluate the diagnostic performance of this new score including IHC, to define the best cut off for inflammatory arthritis recognition, and to compare its diagnostic performance with the KS, considered as the gold standard.

\section{Methods}

\subsection{Patient's samples collection}

Synovial samples from patients were obtained during surgery of arthroplasty or synovectomy with a Ethical Research Agreement (number DC-2011-1399) obtained from the French Ministry of Social Affairs and Health. All patients gave written consent prior surgery. 2.2. Synovial Tissue Immunohistological Staining Synovial tissue was fixed in formalin $4 \%$ for 48 hours, embedded in paraffin and $3 \mu \mathrm{m}$ sectioned (Microtome) as described previously [9]. Sections were deparaffinised in xylene and dehydrated in alcohol and distilled water. Slides were baked for 20 minutes at $97^{\circ} \mathrm{C}$ in 
Citrate (Ki67 slides) or EDTA solution (CD3, CD20, CD68 and CD31) for epitope retrieval. Sections were then incubated with primary mouse antibodies against human CD68, CD3, CD20, Ki67 (Dako, Denmark) and primary rabbit antibodies against human CD31 (Abcam, UK) overnight at $4^{\circ} \mathrm{C}$. Slides were then incubated with biotinylated secondary anti-mouse or anti-rabbit antibodies for 1 hour at room temperature. Immunodetection was performed using streptavidin HRP-complex, revealed with 3,3'-diaminobenyidine (Dako, Denmark). Negative control was analyzed using a similar procedure without primary antibody. Slides were counterstained with Haematoxylin and mounted.

All slides were stained and scored under blinded conditions. Slides were scored by two reviewers independently for Krenn score as previously described [7] and for each immunostaining using a semi-quantitative 4 scale score $(0-3)$ with [ $0=$ no infiltrate], $[1=$ mild infiltrate], [ $2=$ moderate infiltrate], [ $3=$ severe infiltrate], using a pre-defined atlas (Figure 1) [10]. The features were added for a total score out of 24 (Krenn score 0-9 points, and $0-3$ score for each of the 5 immunostainings).

2.3. Statistical analyses

Continuous normally distributed data are presented as the mean with standard deviation (SD) and ordinal non-normally distributed data are presented as the median with interquartile range (IQR). Professional statistician was consulted for statistical design. Differences between groups were analysed using unpaired (2 tailed) non-parametric Mann-Whitney tests. Correlations were assessed using Spearman correlation test. Univariate analysis was performed using logistic regression. Results are presented with Odd Ratio with 95\% confidence intervals. Receptor operating curves (ROC) were performed and area under the curve were calculated and compared using unpaired ( 2 tailed) non parametric Mann-Whitney test. Statistical analysis was performed using SPSS software ${ }^{\circledR}$. P values less than 0.05 were considered significant.

\subsection{Role of the funding source}

The funding sources contributed to the payment of immunohistochemistry material.

\section{Results}

77 patients were included. 45 were females $(58,4 \%)$, mean age was 64.9 years (standard deviation (SD) 12.3 years).

40 had IA reparsed as follows: 31 Rheumatoid arthritis (RA) according to ACR/EULAR criteria [11], 3 had Psoriatic Arthritis (PsA) according to CASPAR criteria [12], 3 had Undifferentiated arthritis (UA), 2 had Spondylarthritis and 1 had Systemic Lupus according to ACR criteria [13]. 77.5\% were treated with csDMARDs (Conventional synthetic Disease Modifying Anti-Rheumatic Drugs) and 25\% with bioDMARDs (Biologic Disease Modifying Anti-Rheumatic Drugs). For RA patients, mean DAS 28 was 3.5 [Standard deviation (SD) 1.22]. "Non inflammatory" arthritis group included 35 patients with Osteoarthritis (OA) and 2 with ligaments or meniscus injuries (Post traumatic Arthritis (PtA)) (Table 1).

Agreement was high between the 2 operators (AN and VK) with a Kappa correlation coefficient of 0.86 [95\% Confidence Interval (CI95) 0.71-0.99].

Mean KS and IMSYC were significantly higher in the IA group ( $p<0.001)$ (Figure 2.A). In univariate analysis by logistic regression, KS (Odd Ratio (OR) 2.9; [CI95 1.8-4.7], CD68 (OR 5.2; [CI95 2.4-11.2]), CD3 (OR 6.9; [CI95 2.9-15.9]), Ki67 (OR 6.4; [CI95 2.9-13.9]), CD31 scores (OR 9.4; [CI95 3.4-26.6]), and CD20 score (OR 2.6; [CI95 1.5-4.6]) were significantly associated with IA.

In the subgroup of RA patients, significant positive correlation was found between Ki67 score and disease activity score (DAS 28) (coefficient correlation " $r "=0.6 ; p=0.045$ ). Correlations coefficients between DAS 28 and CD3 staining ( $\mathrm{r}=0.47 ; \mathrm{p}=0.13), \mathrm{CD} 20$ staining $(\mathrm{r}=0.56 ; \mathrm{p}=0.06)$, CD68 staining $(\mathrm{r}=0.10 ; 0.48), \mathrm{CD} 31$ staining $(\mathrm{r}=0.27 ; \mathrm{p}=0.3)$ were positive but did not reach statistical significance. 
Positive but non-significant correlations were also found between DAS 28 and KS $(\mathrm{r}=0.14 ; \mathrm{p}=0.66)$ or IMSYC $(\mathrm{r}=0.25 ; \mathrm{p}=0.44)$.

ROC curve analysis of diagnostic performances determined the score of 13.5 out of 24 as the best cut off for discrimination between IA and non-IA with a sensitivity of $71.8 \%$ and specificity of $97.3 \%$ (Figure 2.B). With a cut-off of 5.5, KS showed a sensitivity of and a specificity of $66.7 \%$ and $91,9 \%$ for IA discrimination. Area under the curve of IMSYC (0.9574 [CI95 0.921-0.994]) was significantly superior than area under the curve of Krenn Score (0.879 [CI95 0.806-0.952]; $\mathrm{p}=0.0095)$.

\section{Discussion}

In this work we aimed to update Krenn et al. synovitis score by adding 5 relevant immunostainings.

CD68 staining has been widely used in synovitis assessment and is also considered as a biomarker of therapeutic response in RA [14]. Interestingly, the 3 immunomarkers having the highest Odd Ratios were: CD31, CD3 and Ki67. CD31 immunostaining, by recognizing endothelial cells highlights neovascularization. Neovascularization has been shown to play an important pathophysiological role in IA especially in RA [15]. CD3 immunostaining, by detecting $\mathrm{T}$ lymphocytes highlights adaptive immunity cell infiltrate within synovial membrane. Ki67 is an interesting proliferating cell marker, used for tumour diagnosis and prognosis on a regular basis. It has been shown to be overexpressed in inflammatory arthropathies (IA) synovium compared to normal and non-inflammatory arthropathies (NIA) synovium [16]. We also found a good correlation between DAS28 and Ki67 staining in RA patients. The other staining and the Krenn score and IMSYC however showed non-significant positive correlations. This might be due to the relatively small number of synovial membranes in our collection.

KS diagnostic performances were close from the description of the original publication [7]. The ROC analysis showed good performances of the IMSYC for discrimination between IA and NIA. Interestingly, combination of immunostainings or immunostaining tested separately with ROC curve analysis showed lower sensitivity and specificity than the IMSYC (data not shown).

We acknowledge that our work has limitations. The heterogeneous nature of synovial samples, with patients being treated by csDMARDs and bioDMARDs is one of them. We can hypothesize that treatment will reduce inflammation and synovitis score in IA group of patients. However, in our work, clear differences remained in the intensity of inflammation in the IA group. Interestingly, a few OA patients exhibited a higher IMSYC, suggesting an inflammatory pattern. IMSYC could therefore be used for synovial membrane characterization in osteoarthritis with inflammatory features, but this needs to be confirmed by further studies.

This study validates IMSYC diagnostic value in a well-defined collection of synovial sample. This score is relevant as immunophenotype of synovial membrane -especially in RA- can be used as a biomarker for both prognosis and therapeutic decision (tissue-driven therapy) $[17,18]$.

The validation of IMSYC in a well-defined collection of synovial sample is the first step towards a wide acceptance and use for prognosis purposes in translational research.

Acknowledgments:

This work was supported by Inserm, the Arthritis Foundation, Pfizer and the Région Pays de la Loire (Bioregate).

Disclosure of interests: 
The authors declare that they have no competing interest.

\section{References}

[1] Humby F, Kelly S, Bugatti S, Manzo A, Filer A, Mahto A, Fonseca JE, Lauwerys B, D'Agostino MA, Naredo E, Lories R, Montecucco C, Tak PP, Fitzgerald O, Smith MD, Veale DJ, Choy EH, Strand V, Pitzalis C. Evaluation of Minimally Invasive, Ultrasound-guided Synovial Biopsy Techniques by the OMERACT Filter-determining Validation Requirements. J Rheumatol. 2016 ;43(1):208-13.

[2] Najm A, Orr C, Heymann M-F, Bart G, Veale DJ, Le Goff B. Success Rate and Utility of Ultrasound-guided Synovial Biopsies in Clinical Practice. J Rheumatol 2016;43:2113-9.

[3] Orr C, Sousa E, Boyle DL, Buch MH, Buckley CD, Cañete JD, et al. Synovial tissue research: a state-of-the-art review. Nat Rev Rheumatol 2017;13:463-75. doi:10.1038/nrrheum.2017.115.

[4] Pitzalis C, Kelly S, Humby F. New learnings on the pathophysiology of RA from synovial biopsies: Curr Opin Rheumatol 2013;25:334-44.

[5] Townsend MJ. Molecular and cellular heterogeneity in the Rheumatoid Arthritis synovium: clinical correlates of synovitis. Best Pract Res Clin Rheumatol 2014;28:539-49.

[6] Krenn V, Morawietz L, Häupl T, Neidel J, Petersen I, König A. Grading of chronic synovitis - a histopathological grading system for molecular and diagnostic pathology. Pathol-Res Pract 2002;198:317-325.

[7] Krenn V, Morawietz L, Burmester G-R, Kinne RW, Mueller-Ladner U, Muller B, et al. Synovitis score: discrimination between chronic low-grade and high-grade synovitis. Histopathology 2006;49:358-64.

[8] Krenn V, Perino G, Rüther W, Krenn VT, Huber M, Hügle T, et al. 15 years of the histopathological synovitis score, further development and review: A diagnostic score for rheumatology and orthopaedics. Pathol Res Pract 2017;213:874-81.

[9] Boutet M-A, Najm A, Bart G, Brion R, Touchais S, Trichet V, et al. IL-38 overexpression induces anti-inflammatory effects in mice arthritis models and in human macrophages in vitro. Ann Rheum Dis 2017;76:1304-12.

[10] Baeten D, Kruithof E, De Rycke L, Boots, A, Mielants H, Veys, E, et al. Infiltration of the synovial membrane with macrophage subsets and polymorphonuclear cells reflects global disease activity in spondyloarthropathy. Arthritis Res Ther 2005;7:R359-R369.

[11] Alves C, Luime JJ, van Zeben D, Huisman AM, Weel AE, Barendregt PJ, et al. Diagnostic performance of the ACR/EULAR 2010 criteria for rheumatoid arthritis and two diagnostic algorithms in an early arthritis clinic (REACH). Ann Rheum 2011;70:(9)1645-7. [12] Taylor W, Gladman D, Helliwell P, Marchesoni A, Mease P, Mielants H, et al. Classification criteria for psoriatic arthritis: development of new criteria from a large international study. Arthritis Rheum 2006;54:2665-73.

[13] Hochberg MC. Updating the American College of Rheumatology revised criteria for the classification of systemic lupus erythematosus. Arthritis Rheum 1997;40:1725.

[14] Bresnihan B, Pontifex E, Thurlings RM, Vinkenoog M, El-Gabalawy H, Fearon U, et al. Synovial tissue sublining CD68 expression is a biomarker of therapeutic response in rheumatoid arthritis clinical trials: consistency across centers. J Rheumatol 2009;36:1800-2. [15] Fearon U, Griosios K, Fraser A, Reece R, Emery P, Jones PF, et al. Angiopoietins, growth factors, and vascular morphology in early arthritis. J Rheumatol 2003;30:260-8. 
[16] Pessler F, Ogdie A, Diaz-Torne C, Dai L, Yu X, Einhorn E, et al. Subintimal Ki-67 as a synovial tissue biomarker for inflammatory arthropathies. Ann Rheum Dis 2008;67:162-7.

[17] Dennis G, Holweg CT, Kummerfeld SK, Choy DF, Setiadi AF, Hackney JA, et al.

Synovial phenotypes in rheumatoid arthritis correlate with response to biologic therapeutics.

Arthritis Res Ther 2014;16:R90.

[18] Rao DA, Gurish MF, Marshall JL, Slowikowski K, Fonseka CY, Liu Y, et al.

Pathologically expanded peripheral $\mathrm{T}$ helper cell subset drives B cells in rheumatoid arthritis.

Nature 2017;542:110-4.

Table 1. Demographic and clinical data.

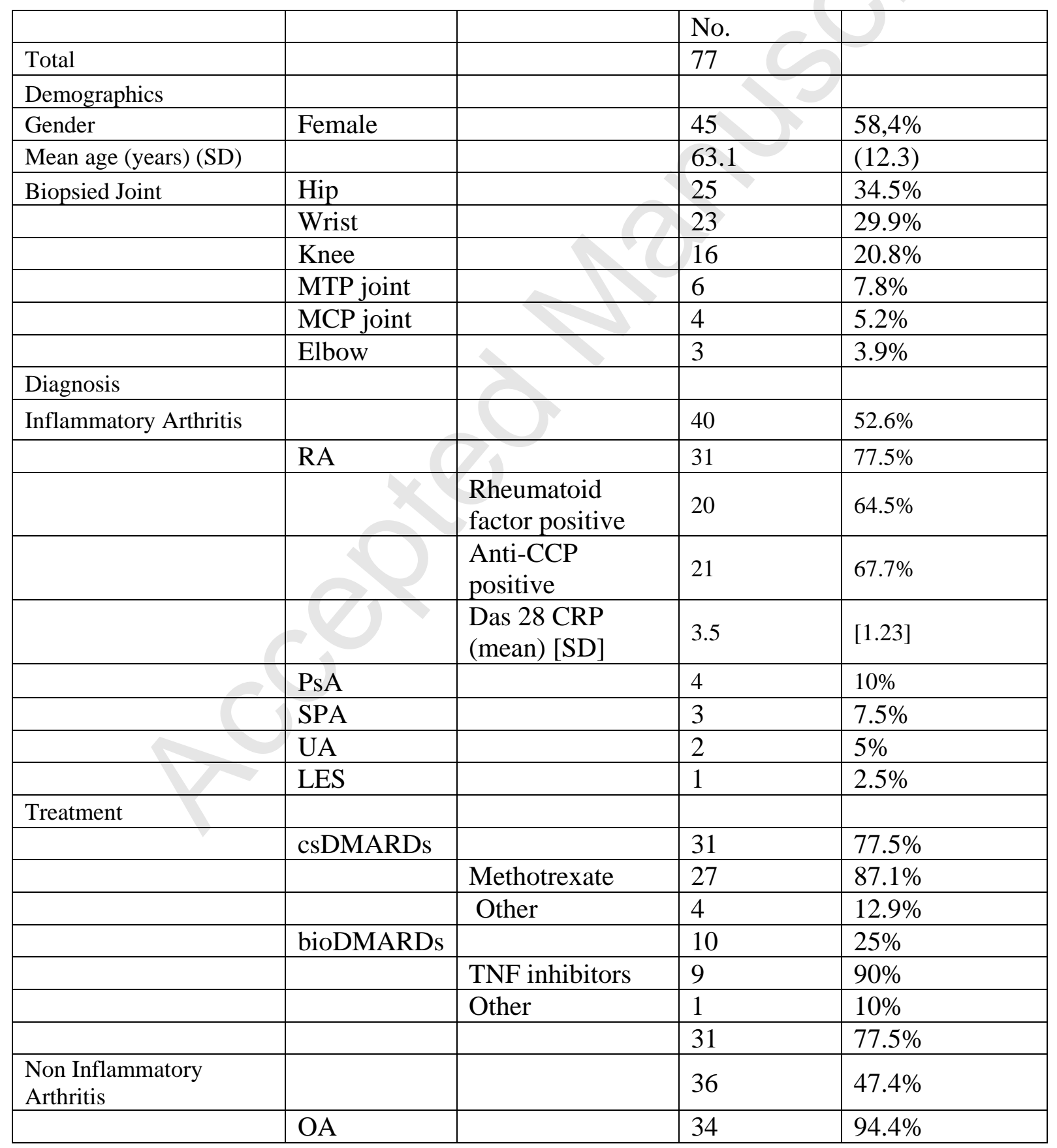




\begin{tabular}{|l|l|l|l|l|}
\hline & PtA & & 2 & $5,6 \%$ \\
\hline
\end{tabular}

RA: Rheumatoid Arthritis, PsA: Psoriatic Arthritis, SPA: Spondyloarthritis, UA:

Undifferentiated arthritis, CRP: C-reactive protein, mg/L: milligrams per liter, csDMARDs: Conventional synthetic Disease Modifying Anti-Rheumatic Drugs, bioDMARDs: Biologic Disease Modifying Anti-Rheumatic Drugs, TNF: Tumor Necrosis Factor, OA: Osteoarthritis, PtA: Post Traumatic Arthritis.

Figure legends

Figure 1: Atlas for semi-quantitative 0-3 scores of the 5 immunostaining CD31, CD3, Ki67, CD68, CD20. Each immunostaining: 0-3 score. Krenn Synovitis Score: 0-9 score. Total Immuno Synovitis Score: 0-24 score.

Figure 2:

A. Krenn Synovitis score and IMmuno SYnovitis sCore in the 2 Inflammatory arthritis and Non Inflammatory arthritis groups. $* *=p<0.0001$

B. ROC curve analysis of Krenn Synovitis score and IMmuno SYnovitis sCore IMSYC.

\section{Highlights}

-IMSYC is a new synovitis score adding 5 immunostainings to Krenn's original synovitis score.

-IMSYC has a better sensitivity and specificity than original synovitis score to discriminate inflammatory and non-inflammatory arthritis.

-IMSYC allows a more accurate synovitis characterization. 